\title{
Compliance of the aorta in two diseases affecting vascular elasticity, familial hypercholesterolemia and diabetes: a MRI study
}

\author{
Sami Soljanlahti' \\ Taina Autti' \\ Laura Hyttinen ${ }^{2}$ \\ Alpo F Vuorio 3 \\ Pekka Keto' \\ Kirsi Lauerma'
}

'Helsinki Medical Imaging Center, Helsinki University Central Hospital, Helsinki, Finland; ' 2 Department of Internal Medicine, North Karelia Central Hospital, Joensuu, Finland; ${ }^{3}$ Division of Internal Medicine, Department of Medicine, University of Helsinki, Helsinki, Finland
Correspondence: Sami Soljanlahti Helsinki Medical Imaging Center, Helsinki University Central Hospital, PL 340, 00029 HUS, Finland

$\mathrm{Tel}+358947174417$

Fax +3589 47I 74404

Email sami.soljanlahti@hus.fi

\begin{abstract}
Arterial elasticity changes in familial hypercholesterolemia $(\mathrm{FH})$ and diabetes mellitus (DM) with different but overlapping mechanisms. We compared aortic elasticity between 19 FH patients with the same mutation, 18 type 2 DM patients, and 30 controls, all aged 48 to 64 . They underwent aortic magnetic resonance imaging, risk-factor assessment, and carotid and femoral ultrasound measurements. All patients were on adequate cardiovascular medication including statins and had established coronary heart disease (CHD). FH patients had longerduration CHD (13.3 \pm 7.7 years) than did DM patients $(5.0 \pm 3.1)$. Aortic compliance in the descending thoracic (DM $0.38 \pm 0.14$ vs control $0.53 \pm 0.19, P=0.032$ ) and abdominal aorta (DM $0.45 \pm 0.20$ vs control $0.66 \pm 0.25, P=0.011$ ) was lower in DM patients than in controls, whereas no significant difference existed between FH patients and controls. Carotid and femoral intima-media thickness was greater in FH and DM patients than in controls with no difference between patient groups. Carotid or femoral plaques appeared in $15(79 \%) \mathrm{FH}$ and in $10(56 \%)$ DM patients. One control had a femoral plaque. Five FH patients showed stenosis, occlusion or both in carotid arteries. In our opinion, DM patients' lower compliance reflect mainly arterial media affecting arteriosclerosis, while FH patients' plaque status and longer duration of CHD suggest more advanced atherosclerosis. The FH patients may therefore be at increased risk for atherothrombotic events. However, due to small patient material, larger confirmatory studies are needed.
\end{abstract}

Keywords: MRI, ultrasound, familial hypercholesterolemia, diabetes mellitus, elasticity, intima-media thickness

\section{Introduction}

Diabetes mellitus (DM) is a common cause of excess mortality, approximately half of which results from cardiovascular diseases (Morgan et al 2000). The prevalence of DM is rising worldwide (Wild et al 2004) and causing growing expenses for the health care system in addition to life-years lost.

Another disease causing excess cardiovascular mortality is familial hypercholesterolemia (FH) (Anonymous 1999). In FH, a hereditary low-density lipoprotein (LDL) receptor defect results, from birth, in impaired hepatic cholesterol uptake, which in heterozygotes leads to 2- to 3-fold higher blood cholesterol levels than normal. This results in accelerated atherosclerosis. The prevalence of heterozygous FH is $1 / 500$ worldwide (Goldstein et al 2001).

Both DM and FH are risk factors for atherosclerosis, which is associated with decreased arterial elasticity (van Popele et al 2001) in addition to plaque formation. Endothelial dysfunction, inflammation, fibrosis, and calcification of the arterial intima are the proposed mechanisms. Another mechanism leading to reduction in arterial elasticity is arteriosclerosis. This term associated with DM and aging refers 
to degradation of elastin, proliferation of collagen, and deposition of calcium in the media and adventitia in contrast to changes in the intima in atherosclerosis (Safar 2007). These two mechanisms for loss of arterial elasticity often overlap (Safar 2007), however, and the terms are sometimes confusingly interchanged.

Atherosclerosis of different vascular beds show positive association (Sternby 1968) and magnetic resonance imaging (MRI) is an established method to study vessel wall elasticity (Oliver and Webb 2003). In our previous study on young heterozygous $\mathrm{FH}$ patients carotid intima-media thickness (IMT) correlated with aortic elasticity markers (pulse wave velocity and complience) assessed with MRI (Soljanlahti et al 2008).

Careful risk factor treatment, statins in particular (Smilde et al 2001), may slow down the atherosclerotic process or cause even regression of atherosclerotic lesions. The results of our earlier MRI studies on young (6 to 48 years of age) heterozygous FH patients and healthy controls are in line with this showing no difference between groups in aortic morphology and function (Soljanlahti et al 2008) or brain lesions of vascular origin (Soljanlahti et al 2005). The purpose of the present study was to assess the elastic properties of aorta with MRI in patients with more advanced FH disease. The results were compared with DM, in which aortic compliance is decreased due to arterio- and atherosclerosis (Safar 2007). We also compared the aortic data to the carotid and femoral IMT, a measure of overall atherosclerosis, assessed with ultrasound (US).

\section{Material and methods}

\section{Patients}

The patient register of the North Karelia Central Hospital (Joensuu, Finland) contained data on $30 \mathrm{FH}$ patients 48 to 64 years of age who had the same DNA-verified (Koivisto et al 1993) North Karelia (NK) mutation in year 2003. The mutation produces similar phenotype (tendon xanthomas, 2-3-fold elevation in LDL cholesterol) like other receptor defective FH mutations. They also had coronary heart disease (CHD) verified from medical records and by physical examination. Eight of them were excluded because 3 also had DM, 1 had a body mass index (BMI) of 46, 2 had an unreliable diagnosis of CHD, 1 was unable to travel, and 1 was unreachable. Of the remaining 22 patients, 19 agreed to participate.

The same CHD criteria and the same patient register as for FH-NK patients was used to find 22 sex- and age-matched type 2 DM patients who had been treated at the department of internal medicine of the North Karelia Central hospital. Two were excluded because they did not use statin medication. Of the remaining 20 patients, 18 (aged 51-64) agreed to participate.

All FH-NK and DM patients used statin medication. See Table 1 for their cardiovascular medication and

Table I Characteristics and cardiovascular medication of controls, DM, and FH-NK patients

\begin{tabular}{|c|c|c|c|c|}
\hline & $\begin{array}{l}\text { DM } \\
(n=18)\end{array}$ & $\begin{array}{l}\text { FH-NK } \\
(n=19)\end{array}$ & $\begin{array}{l}\text { Controls } \\
(n=29)\end{array}$ & $P$-value \\
\hline Males/females, no. & $1 \mathrm{I} / 7$ & $11 / 8$ & $13 / 16$ & \\
\hline Age, $y$ & $57.9 \pm 3.7$ & $55.9 \pm 4.5$ & $56.0 \pm 3.8$ & 0.210 \\
\hline $\mathrm{BMI}, \mathrm{kg} / \mathrm{m}^{2}$ & $32.6 \pm 4.8$ & $26.7 \pm 2.8$ & $25.9 \pm 3.5$ & $\begin{array}{l}0.000(\mathrm{DM}>\mathrm{FH} ; \\
\mathrm{DM}>\mathrm{CTRL} ; \\
\mathrm{FH}=\mathrm{CTRL})\end{array}$ \\
\hline Systolic blood pressure, $\mathrm{mmHg}$ & $143 \pm 21$ & $137 \pm 23$ & $130 \pm 14$ & 0.081 \\
\hline Diastolic blood pressure, $\mathrm{mmHg}$ & $83 \pm 13$ & $78 \pm 10$ & $79 \pm 9$ & 0.179 \\
\hline Statin usage, $y$ & $5.2 \pm 2.9$ & $15.1 \pm 1.3$ & 0 & $0.000^{\mathrm{a}}$ \\
\hline Duration of CHD, y & $5 \pm 3.1$ & $13.3 \pm 7.6$ & 0 & $0.000^{\mathrm{a}}$ \\
\hline Statins, users & 18 & 19 & 0 & \\
\hline Beta blockers, users & 18 & 18 & I & \\
\hline Acetylsalicylic acid, users & 16 & 17 & 0 & \\
\hline ARB or ACE inhibitors, users & II & 3 & 0 & \\
\hline Smokers & 3 & 3 & 0 & \\
\hline
\end{tabular}

Notes: Values are means \pm SD unless otherwise stated. ${ }^{\mathrm{F}} \mathrm{FH}$ and DM groups compared.

Abbreviations: DM, diabetes mellitus; FH-NK, familial hypercholesterolemia-North Karelia; BMI, body mass index; CHD, coronary heart disease; ACE, angiotensin-converting enzyme; ARB, angiotensin-receptor blockers. 
characteristics. All FH-NK patients and 17 of the $18 \mathrm{DM}$ patients had had AMI, a coronary bypass operation, or balloon angioplasty. Two DM and two FH-NK patients had each had transient ischemic attack (TIA), and one FH-NK patient had a history of ischemic stroke. No other patients had any history of neurological diseases.

The control group comprised 30 controls (aged 49-63) recruited among acquaintances of the Helsinki Medical Imaging Center (Helsinki, Finland) staff. None of the controls had CHD, DM, or history of any vascular or neurological disease. One control was excluded because of Moya-Moya disease detected in brain MRI.

A medical ethics committee approved the study with informed consent obtained from all subjects.

\section{MRI}

MRI was performed with a $1.5 \mathrm{~T}$ Siemens Sonata imager (Erlangen, Germany) with a phased array coil. A breath-hold oblique sagittal TSE sequence (TR/ TE/ Flip Angle/ FOV/ Matrix/ Slice Thickness $=47 / 1.6 / 65 / 298 * 340 / 148 * 256 / 6$ ) of the aorta was taken to assess the imaging planes for the cine sequence (TR/ TE/ Flip Angle/ FOV/ Matrix /Slice Thickness $=28 / 3.2 / 30 / 223 * 340 / 156 * 256 / 5$ ) taken at the level of the right pulmonary artery and at the level of the renal artery. Time resolution was $28 \mathrm{~ms}$.

Electrocardiogram gating was used with all sequences. Due to poor image quality, one DM patient and one control were excluded from analysis of compliance of the ascending aorta and descending thoracic aorta, and one FH patient was excluded from descending abdominal aorta measurement.

The images were analyzed with Siemens Leonardo workstation's Argus software (Erlangen, Germany) by S.S. who was blinded to the clinical and laboratory data and had 5 years of experience with the measurements. Compliance $(\% / \mathrm{mmHg})$ was calculated from the cine images by the equation: ([Amax-Amin]/Amin*100)/(Ps-Pd), where Amax $=$ maximum luminal area, Amin $=$ minimal luminal area, $\mathrm{Ps}=$ systolic blood pressure, and $\mathrm{Pd}=$ diastolic blood pressure (Mohiaddin et al 1989).

\section{Ultrasound}

One experienced radiologist (K.L.) performed all ultrasound examinations with an 8-12 Mhz linear transducer. The subjects were examined in the supine position. IMT was measured with high-resolution B-mode (Pignoli et al 1986) for the far wall of each common carotid artery $1 \mathrm{~cm}$ below the bifurcation, and of the left femoral artery at the level of the inguinal ligament, and the mean of 3 consecutive measurements was calculated. The carotid bifurcations and the left femoral artery were examined for plaques. Doppler flow measurements were performed to assess the severity of arterial stenoses caused by the plaques. Stenosis of $70 \%$ or more was considered significant (Grant et al 2003). Ultrasound data for two controls were unavailable.

\section{Laboratory tests}

After overnight fasting, each subject provided a venous blood sample. Serum total cholesterol, triglycerides, high-density lipoprotein (HDL) cholesterol, fasting glucose, glycosylated hemoglobin, homocysteine, liproprotein a, insulin, and highsensitivity C-reactive protein (hsCRP) were determined by standard methods. The LDL cholesterol value was calculated by the Friedewald formula. To take into account each participant's lifelong cholesterol burden, we calculated the cholesterol-years score, as suggested by Hoeg and colleagues (1994). Blood pressure was measured twice with an automatic meter from the left arm of the patient lying supine, with the mean of two measurements used in the calculations. One control was excluded from laboratory assessments because her anti-inflammatory therapy was started between the MR examination and laboratory tests. Because infections can elevate CRP level beyond that associated with inflammation in cardiovascular diseases (Ridker 2003), 4 DM patients whose hsCRP levels exceeded $10 \mathrm{mg} / \mathrm{L}$ were thus excluded from CRP analysis.

\section{Statistical methods}

Variables between the groups (controls, FH, and DM patients) were compared with ANOVA or the Kruskal-Wallis test, as appropriate. A $P$-value $<0.05$ was considered statistically significant.

\section{Results MRI}

DM patients had significantly less compliant descending thoracic and abdominal aorta than did controls, but this difference did not reach significance in ascending aorta. No significant difference existed between FH-NK and DM patients (Figure 1).

\section{Ultrasound}

In all vessels, IMT appeared significantly greater in the FH-NK and DM patients than in the controls (Figure 2). No significant differences appeared between FH-NK and DM patients. Carotid or femoral plaques appeared in $15(79 \%)$ FH-NK and in 10 (56\%) DM patients. Only one control had a plaque, and it was in femoral artery (Figure 3). Five 


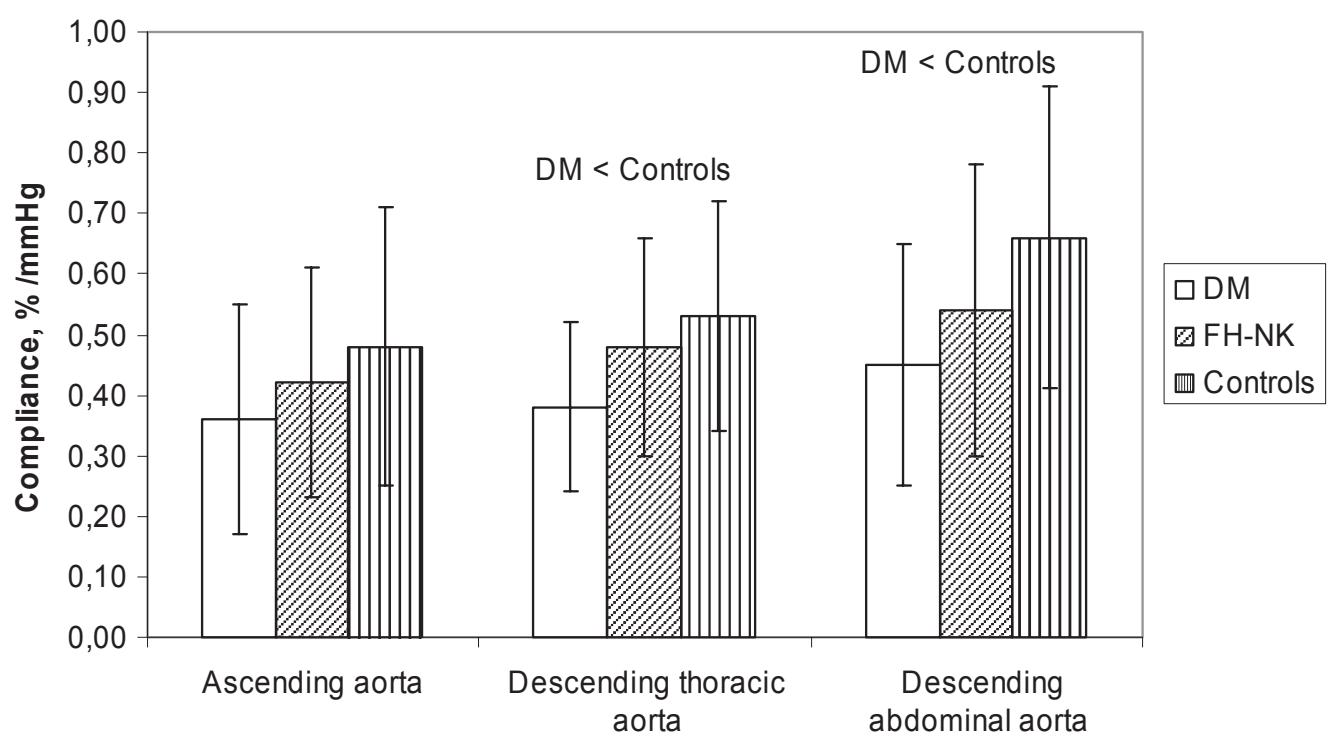

Figure I Aortic Compliance.

Note: Bars indicate mean, whiskers \pm SD.

Abbreviations: DM, diabetes mellitus; FH-NK, familial hypercholesterolemia-North Karelia; SD, standard deviation.

FH patients showed stenosis, occlusion or both in carotid arteries: four showed stenosis, the left internal carotid artery was occluded in one, and another had occlusion of the right internal carotid artery. None of the DM patients or controls showed significant stenosis or occlusion.

\section{Laboratory tests}

Cholesterol-years score and total and LDL cholesterol level of the FH-NK patients were greater than those of DM patients and controls. DM patients had higher trigycerides, fasting glucose, insulin, percentage of glycosylated hemoglobin, homocysteine, and hsCRP than did FH-NK patients and controls (Table 2).

\section{Discussion}

Compliance of the descending aorta was lowest in the DM patients and highest in controls, but no significant difference emerged between FH and DM patients. Carotid and femoral

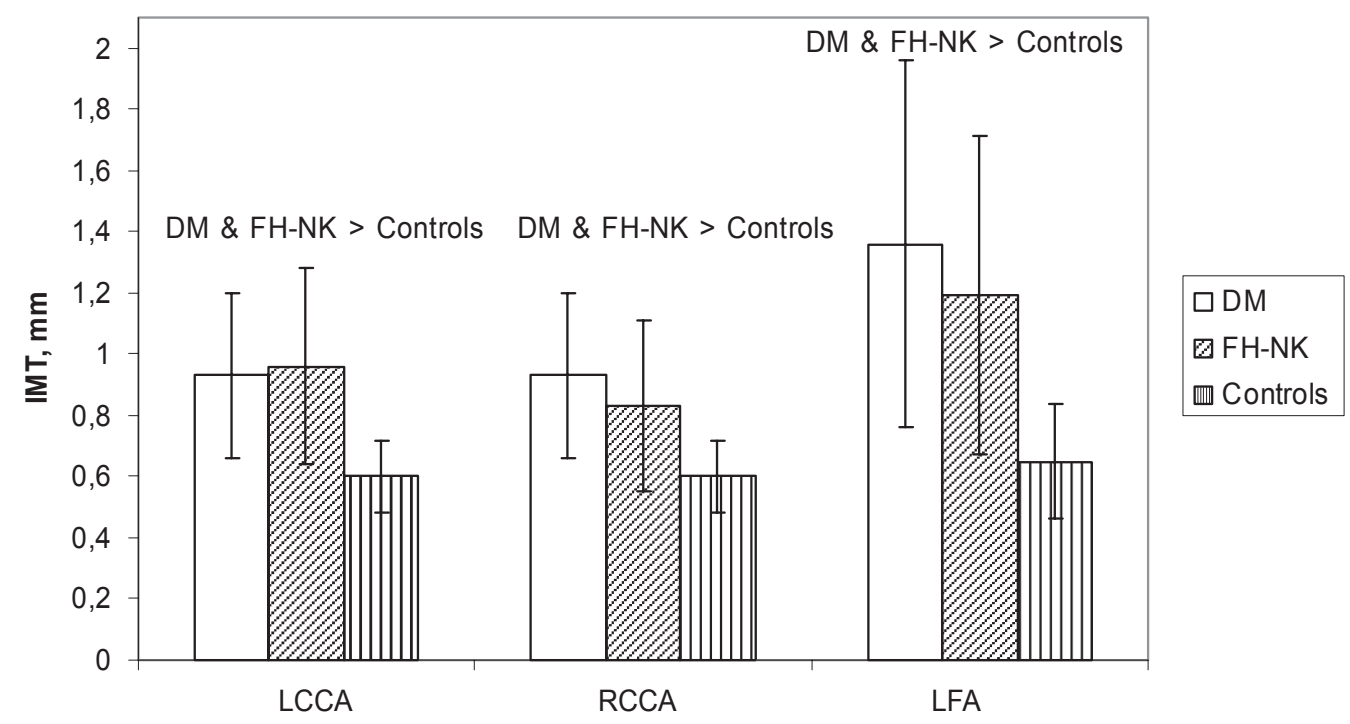

Figure 2 Carotid and Femoral IMT.

Note: Bars indicate mean, whiskers \pm SD.

Abbreviations: IMT, intima-media thickness; FH-NK, familial hypercholesterolemia North Karelia; DM, diabetes mellitus; LCCA, left common carotid artery; RCCA, right common carotid artery; LFA, left femoral artery. 


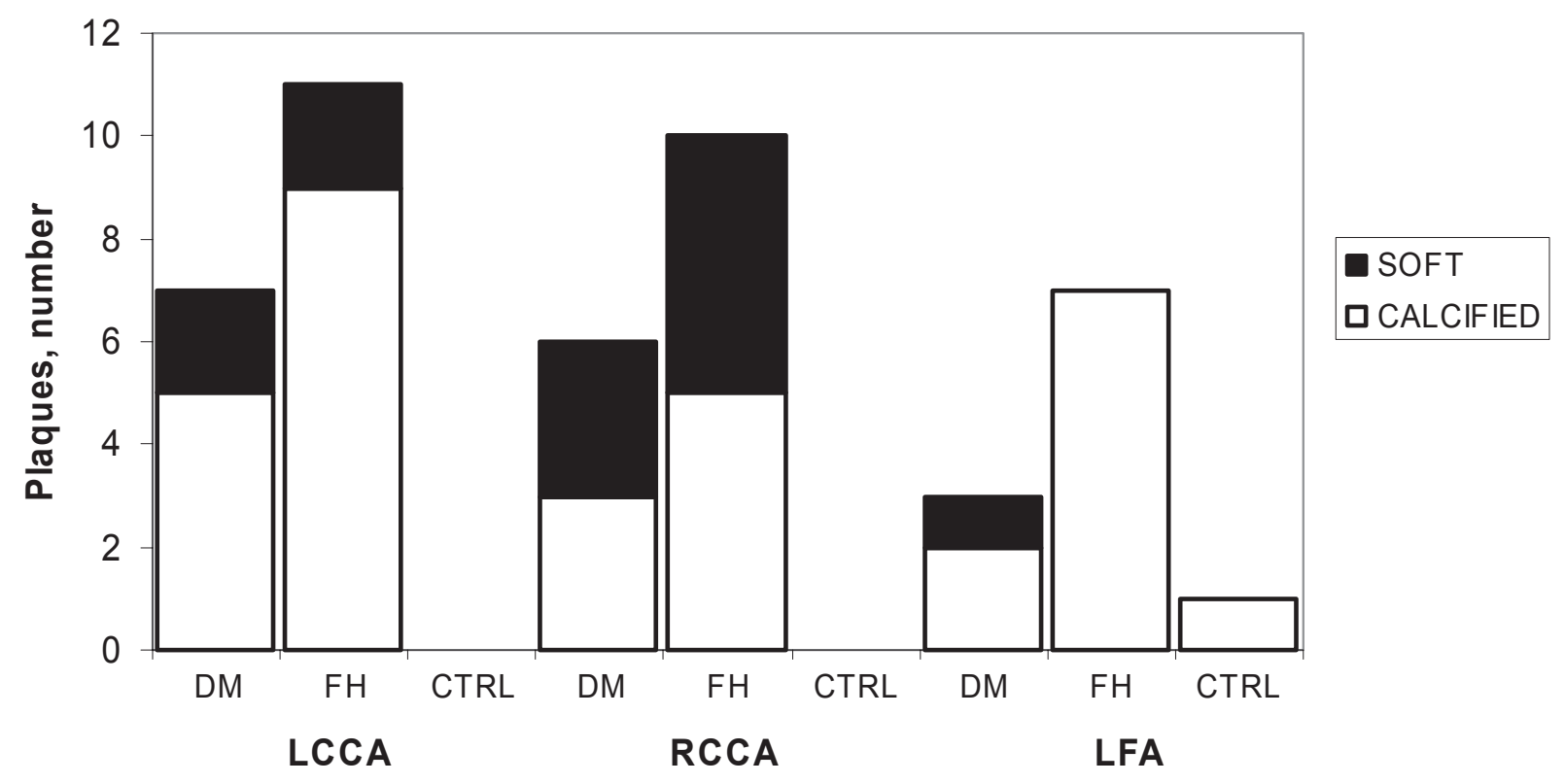

Figure 3 Carotid and Femoral Plaques.

Abbreviations: LCCA, left common carotid artery; RCCA, right common carotid artery; LFA, left femoral artery; DM, diabetes mellitus; FH, familial hypercholesterolemia; CTRL, controls.

IMT of DM and FH patients was greater than that of the controls, with no difference between IMT of FH and DM patients. Only the FH patients had significant carotid stenoses or occlusions; they also had more plaques than either DM patients or controls.

Our obese DM patients had compliance of the descending aorta lower than that of controls. This is in concordance with a recent MRI study showing lower aortic elasticity than in their 16 controls in 14 type $2 \mathrm{DM}$ patients averaging 55 years of age and without any complications (van der Meer et al 2007). In addition, decreased aortic elasticity appeared in the MRI of 21 healthy obese (BMI > 30) men compared to 25 lean (BMI 19-25) controls (Danias et al 2003), all 20 to 40 years old. The aortic compliance of our FH patients was lower than in controls, although their difference was not significant. This is in line with an aortic MRI study on 10 relatively young ( 34 years old on average) asymptomatic heterozygous FH patients with no difference in compliance compared to that of controls (Toikka et al 1999) and with our earlier study on healthy 6- to 48-year-old FH patients with no difference in aortic compliance compared to that of controls (Soljanlahti et al 2008). Our FH patients with verified CHD were expected to have decreased aortic compliance, as CHD is associated with lower aortic elasticity (Mohiaddin et al 1989; Forbat et al 1998). Their improved risk-factor treatment, statin treatment in particular, may have had beneficial effects on compliance, an effect shown in one MRI study of 64 hypercholesterolemic patients (Forbat et al 1998).

The carotid and femoral IMT of our DM and FH patients was higher than that of the controls as expected. Our FH patients had more carotid and femoral plaques than did DM patients or controls. Only the FH patients had occlusions and significant stenoses of carotid arteries suggesting the most advanced atherosclerosis. In the atherosclerotic process, an increase in IMT precedes plaque formation (Zureik et al 2000). While statins cause regression of atherosclerotic lesions (Smilde et al 2001) and calcifications (Callister et al 1998; Williams et al 1998), to our knowledge, information is lacking on their effect on severely stenosed arteries. CHD duration was longer in our FH than DM patients (Table 1). The reason for a higher number of plaques in our $\mathrm{FH}$ patients may be their long exposure to high cholesterol concentrations, before statin treatment in the late 1980's became available. In addition, the current cholesterol levels of our FH patients exceeded those of the DM patients despite statin treatment (Table 2). Furthermore, before statin treatment, FH patients showed a risk for strokes over 20-fold compared to general population (Kaste and Koivisto 1988). Statins provide effects beyond cholesterol lowering (Miida et al 2007), ones which may stabilize atherosclerotic plaques and prevent strokes and other atherothrobotic events. Our FH patients showed no more brain lesions than did controls (Soljanlahti et al 2007). 
Table 2 Laboratory data on controls and DM, and FH-NK patients

\begin{tabular}{|c|c|c|c|c|}
\hline & $\operatorname{DM}(n=18)$ & FH-NK $(n=19)$ & Controls $(n=28)$ & P-value \\
\hline Total cholesterol, $\mathrm{mmol} / \mathrm{L}$ & $4.7 \pm 0.9$ & $6.0 \pm 1.0$ & $5.6 \pm 0.8$ & $0.000(\mathrm{DM}<\mathrm{FH} ; \mathrm{DM}<\mathrm{CTRL} ; \mathrm{FH}=\mathrm{CTRL})$ \\
\hline LDL cholesterol, mmol/L & $2.5 \pm 0.7$ & $4.1 \pm 0.9$ & $3.3 \pm 0.7$ & 0.000 (DM < FH; DM < CTRL; FH > CTRL) \\
\hline HDL cholesterol, mmol/L & $1.2 \pm 0.2$ & $1.4 \pm 0.3$ & $1.7 \pm 0.4$ & 0.000 (DM = FH; DM $<$ CTRL; FH $<\mathrm{CTRL})$ \\
\hline Triglycerides, mmol/L & $2.1 \pm 0.9$ & $1.2 \pm 0.4$ & $1.4 \pm 0.6$ & $0.00 \mathrm{I}(\mathrm{DM}>\mathrm{FH} ; \mathrm{DM}>\mathrm{CTRL} ; \mathrm{FH}=\mathrm{CTRL})$ \\
\hline Cholesterol-years score, mmol-y/L & $368 \pm 89$ & $516 \pm 96$ & $313 \pm 58$ & $0.000(\mathrm{DM}<\mathrm{FH} ; \mathrm{DM}=\mathrm{CTRL} ; \mathrm{FH}>\mathrm{CTRL})$ \\
\hline Fasting glucose, $\mathrm{mmol} / \mathrm{L}$ & $8.7 \pm 3$ & $5.2 \pm 0.6$ & $5.1 \pm 0.4$ & 0.000 (DM > FH; DM > CTRL; FH = CTRL) \\
\hline Insulin, mU/L & $3 I .1 \pm 31.4$ & $7.5 \pm 3.7$ & $6 \pm 4.5$ & $0.000(\mathrm{DM}>\mathrm{FH} ; \mathrm{DM}>\mathrm{CTRL} ; \mathrm{FH}=\mathrm{CTRL})$ \\
\hline Glycosylated hemoglobin, \% & $8.2 \pm 1.6$ & $5.7 \pm 0.2$ & $5.9 \pm 0.3$ & 0.000 (DM $>$ FH; DM $>$ CTRL; FH = CTRL) \\
\hline Lipoprotein a, g/L & $0.22 \pm 0.21$ & $0.20 \pm 0.21$ & $0.21 \pm 0.20$ & 0.359 \\
\hline Homocysteine, $\mu \mathrm{mol} / \mathrm{L}$ & $15 \pm 3$ & $12.2 \pm 2.5$ & $13.1 \pm 4.8$ & 0.047 (DM > FH; DM = CTRL; FH = CTRL) \\
\hline $\mathrm{HsCRP}, \mathrm{mg} / \mathrm{L}$ & $3.07 \pm 2.45$ & $2.22 \pm 2.34$ & $1.35 \pm 1.92$ & $0.00 \mathrm{I}(\mathrm{DM}>\mathrm{FH} ; \mathrm{DM}>\mathrm{CTRL} ; \mathrm{FH}=\mathrm{CTRL})$ \\
\hline
\end{tabular}

Note: Values are means \pm SD.

Abbreviations: DM, diabetes mellitus; FH-NK, familial hypercholesterolemia-North Karelia; CTRL, control; LDL, low-density lipoprotein; HDL, high-density lipoprotein; HsCRP, high-sensitivity C-reactive protein; SD, standard deviation.

The major limitation of our study is the relatively small number of patients and controls. This may explain why no significant difference was found in compliance between $\mathrm{FH}$ patients compared to DM patients and controls.

We conclude that compliance of the descending aorta was lowest in DM patients and highest in controls, with no significant difference between FH and DM patients. Carotid and femoral IMT was greater in FH and DM patients than in controls. FH patients had more plaques and were the only ones with stenoses in carotid arteries. We theorize that the differences between FH and DM reflect the differences between athero- and arteriosclerosis. Furthermore, the plaque status of $\mathrm{FH}$ patients suggests that they are probably more prone to thromboembolic complications of atherosclerosis than are DM patients; their statin treatment, however, may lower the risk. Due to small sample size, larger studies are needed to confirm the findings since arterio- and atherosclerosis are overlapping processes.

\section{Acknowledgments and funding}

Supported by the grants from the Paulo, P.O Klingendahl, and Finnish Cultural Foundations and Schering research foundation, the Radiological Society of Finland, and the Helsinki University Central Hospital Research Funds (TYH 1216, TYH 3229). The authors wish to thank the FH-NK and DM patients and the controls for participation and Timo Päivärinta and Aki Syrjälä for technical assistance. The authors report no conflicts of interest.

\section{References}

Anonymous. 1999. Mortality in treated heterozygous familial hypercholesterolaemia: implications for clinical management. Scientific Steering Committee on behalf of the Simon Broome Register Group. Atherosclerosis, 142:105-12.
Callister TQ, Raggi P, Cooil B, et al. 1998. Effect of HMG-CoA reductase inhibitors on coronary artery disease as assessed by electron-beam computed tomography. $N$ Engl J Med, 339:1972-8.

Danias PG, Tritos NA, Stuber M, et al. 2003. Comparison of aortic elasticity determined by cardiovascular magnetic resonance imaging in obese versus lean adults. Am J Cardiol, 91:195-9.

Forbat SM, Naoumova RP, Sidhu PS, et al. 1998. The effect of cholesterol reduction with fluvastatin on aortic compliance, coronary calcification and carotid intimal-medial thickness: a pilot study. J Cardiovasc Risk, 5:1-10.

Goldstein JL, Hobbs HH, Brown MS. 2001. Familial hypercholesterolemia. In: Scriver CR, Beaudet AL, Sly WS (eds.). The metabolic and molecular bases of inherited diseases. New York: Mc Graw-Hill Book Co., pp. 2863-913.

Grant EG, Benson CB, Moneta GL, et al. 2003. Carotid artery stenosis: gray-scale and Doppler US diagnosis - Society of Radiologists in Ultrasound Consensus Conference. Radiology, 229:340-6.

Hoeg JM, Feuerstein IM, Tucker EE. 1994. Detection and quantitation of calcific atherosclerosis by ultrafast computed tomography in children and young adults with homozygous familial hypercholesterolemia. Arterioscler Thromb, 14:1066-74.

Kaste M, Koivisto P. 1988. Risk of brain infarction in familial hypercholesterolemia. Stroke, 19:1097-100.

Koivisto U-M, Hamalainen L, Taskinen M-R, et al. 1993. Prevalence of familial hypercholesterolemia among young north Karelian patients with coronary heart disease: a study based on diagnosis by polymerase chain reaction. J Lipid Res, 34:269-77.

Miida T. Takahashi A. Ikeuchi T. 2007. Prevention of stroke and dementia by statin therapy: experimental and clinical evidence of their pleiotropic effects. Pharmacol Ther, 113:378-93.

Mohiaddin RH, Underwood SR, Bogren HG, et al. 1989. Regional aortic compliance studied by magnetic resonance imaging: the effects of age, training, and coronary artery disease. Br Heart J, 62:90-6.

Morgan CL, Currie C, Peters JR. 2000. Relationship between diabetes and mortality: A population study using record linkage. Diabetes Care, 23:1103-7.

Oliver JJ, Webb DJ. 2003. Noninvasive assessment of arterial stiffness and risk of atherosclerotic events. Arterioscler Thromb Vasc Biol, 23:554-66.

Pignoli P, Tremoli E, Poli A, et al. 1986. Intimal plus medial thickness of the arterial wall: a direct measurement with ultrasound imaging. Circulation, 74:1399-406.

Ridker PM. 2003. Clinical applications of C-reactive protein for cardiovascular disease detection and prevention. Circulation, 107:363-9. 
Safar ME. 2007. Arterial stiffness: a simplified overview in vascular medicine. Adv Cardiol, 44:1-18.

Smilde TJ, van Wissen S, Wollersheim H, et al. 2001. Effect of aggressive versus conventional lipid lowering on atherosclerosis progression in familial hypercholesterolaemia (ASAP): a prospective, randomised, double-blind trial. Lancet, 357:577-81.

Soljanlahti S, Autti T, Lauerma K, et al. 2005. Familial hypercholesterolemia patients treated with statins at no increased risk for intracranial vascular lesions despite increased cholesterol burden and extracranial atherosclerosis. Stroke, 36:1572-4.

Soljanlahti S, Raininko R, Hyttinen L, et al. 2007. Statin-treated familial hypercholesterolemia patients with coronary heart disease and pronounced atherosclerosis do not have more brain lesions than healthy controls in later middle age. Acta Radiol, 48:894-9.

Soljanlahti S, Autti T, Vuorio AF, et al. 2008. Aorta of young and middleaged heterozygous familial hypercholesterolemia patients shows no functional or morphological impairment assessed by MRI. Vasc Health Risk Manag, 4(4):In press.

Sternby NH. 1968. Atherosclerosis in a defined population: an autopsy survey in Malmö, Sweden. Acta Pathol Microbiol Scand, (Suppl) 194:3-216.
Toikka JO, Niemi P, Ahotupa M, et al. 1999. Large-artery elastic properties in young men: relationships to serum lipoproteins and oxidized lowdensity lipoproteins. Arterioscler Thromb Vasc Biol, 19:436-41.

van der Meer RW, Diamant M, Westenberg JJ, et al. 2007. Magnetic resonance assessment of aortic pulse wave velocity, aortic distensibility, and cardiac function in uncomplicated type 2 diabetes mellitus. J Cardiovasc Magn Res, 9:645-51.

van Popele NM, Grobbee DE, Bots ML, et al. 2001. Association between arterial stiffness and atherosclerosis: the Rotterdam study. Stroke, 32:454-60.

Wild S, Roglic G, Green A, et al. 2004. Global prevalence of diabetes: Estimates for the year 2000 and projections for 2030. Diabetes Care, 27:1047-53

Williams JK, Sukhova GK, Herrington DM, et al. 1998. Pravastatin has cholesterol - lowering independent effects on the artery wall of atherosclerotic monkeys. J Am Coll Cardiol, 31:684-91.

Zureik M, Ducimetière P, Touboul P-J, et al. 2000. Common carotid intimamedia thickness predicts occurrence of carotid atherosclerotic plaques: longitudinal results from the aging vascular (EVA) study. Arterioscler Thromb Vasc Biol, 20:1622-9. 
